\title{
Optimum Response Filter Setting for Air Conduction- Induced Ocular Vestibular Evoked Myogenic Potential
}

DOI: $10.3766 /$ jaaa.17098

\author{
Niraj Kumar Singh* \\ Kumaran Thirunavukkarasu* \\ Animesh Barman*
}

\begin{abstract}
Background: A wide range of normative values of amplitude and latencies can be noticed in the publications on ocular vestibular evoked myogenic potential (oVEMP), possibly because of the inconsistent use of various stimulus and acquisition-related parameters such as response filter, gaze angle, onset polarity of stimulus, etc. One major nonuniform parameter across studies is the response filter. Several band-pass response filters such as $0.5-500,1-1000,5-500,5-800,10-750,20-2000,100-3000$, and 200-1000 Hz have been used across published studies, and a wide range of normative values can be noticed. However, there is paucity of literature evidence to show that variations in response filters could cause alterations in oVEMP response.
\end{abstract}

Purpose: This study aimed to investigate the effects of changes in response filter setting on oVEMP.

Research Design: Normative study using repeated measures research design.

Study Sample: Young adults in the age range of $18-35$ years $(N=150)$ and older adults in the age range of $60-70$ years $(\mathrm{N}=10)$.

Intervention: Contralateral air conduction oVEMP.

Data Collection and Analysis: Contralateral air conduction oVEMP was obtained from only one ear of all participants. Low-pass filters (LPFs) of 500, 700, 1000, 1500, 2000, and $3000 \mathrm{~Hz}$ and high-pass filters (HPFs) of $0.1,1,10$, and $30 \mathrm{~Hz}$ were used in all possible combinations of one LPF and one HPF to create band-pass filters. Latencies, peak-to-peak amplitude, and signal-to-noise ratio (SNR) were obtained for each response and comparison was made between various band-pass filters.

Results: In young adults, there was a significant reduction in $\mathrm{n} 1$ and $\mathrm{p} 1$ latencies with increasing HPF and LPF $(p<0.01)$ and a significant reduction in peak-to-peak amplitude with increasing $\operatorname{HPF}(p<0.008)$. The peak-to-peak amplitude was significantly not affected by changes in LPF $(p>0.05)$. In older adults, the response rate was better for $0.1-$ to $1000-\mathrm{Hz}$ than 1 - to $1000-\mathrm{Hz}$ band-pass filters.

Conclusions: The optimum band-pass filter is $0.1-1000 \mathrm{~Hz}$ for recording oVEMP as it produces the largest amplitude oVEMP without compromising on SNR and causes improved response rate in older adults compared with 1 - to $1000-\mathrm{Hz}$ filters. Therefore, clinical recording of oVEMP should use $0.1-1000 \mathrm{~Hz}$ for obtaining large amplitude potentials and improving the chances of response detection in clinical population.

Key Words: air conduction oVEMP, older adults, response filter set, utricle

Abbreviations: ANOVA = analysis of variance; EMG = electromyography; HPF = high-pass filter; LPF = low-pass filter; oVEMP = ocular vestibular evoked myogenic potentials; SNR = signal-to-noise ratio; VEMP $=$ vestibular evoked myogenic potential

*Department of Audiology, All India Institute of Speech and Hearing, Mysore, India

Corresponding author: Niraj Kumar Singh, Department of Audiology, All India Institute of Speech and Hearing, Mysore 570006, India; Email: niraj6@gmail.com

This paper is an outcome of a project funded by AIISH Research Fund, Ministry of Health and Family Welfare, Government of India (reference: SH/CDN/ARF-05/2014-15). We thank the Ministry of Health and Family Welfare, Government of India, for funding the project. 


\section{INTRODUCTION}

$\mathrm{V}$ estibular evoked myogenic potentials (VEMPs) are otolith-initiated muscle responses (Colebatch and Halmagyi, 1992). When recorded from the extraocular muscles, the VEMP response is referred as ocular VEMP (oVEMP) (Rosengren et al, 2005; Chihara et al, 2007; Todd et al, 2007; Singh and Barman, 2013; Singh et al, 2014).

The effects of changes in several stimulus parameters such as intensity (Murnane et al, 2011), frequency (Chihara et al, 2009; Singh and Barman, 2013; 2014; 2016a,b), stimulus type (Todd et al, 2007; Curthoys, 2010), stimulus duration (Cheng et al, 2012; Lim et al, 2013; Kantner et al, 2014), and repetition rate (Singh et al, 2014) on oVEMP responses were explored and as a result, the optimum values of these parameters were suggested for the clinical recording of oVEMP. Likewise, the response acquisition-related parameters such as electrode positioning (Murnane et al, 2011; Sandhu et al, 2013) and degree of gaze elevation (Govender et al, 2009; Murnane et al, 2011; Rosengren et al, 2013) were explored and optimum values were recommended. The response filter setting [low-pass filter (LPF) and high-pass filter (HPF)] is among the most important acquisition-related parameters affecting all acoustically evoked potentials (Cacace et al, 1980; Goodin et al, 1992) and oVEMP should be no different.

Studies on oVEMP have used several different response filter settings. Although most studies have used an LPF of $1000 \mathrm{~Hz}$ and an HPF of $1 \mathrm{~Hz}$ (Murnane et al, 2011; Piker et al, 2011; Singh and Barman, 2013; 2014; 2015), some of the others have used band-pass filters of $5-500 \mathrm{~Hz}$ (Chihara et al, 2009; Seo et al, 2013), 5-800 $\mathrm{Hz}$ (Walther and Blodow, 2013), 10-750 Hz (Jerin et al, 2014), 20-2000 Hz (Nguyen et al, 2010), 100-3000 Hz (El-Mahallawi et al, 2012), and 200-1000 Hz (Gozke et al, 2010). This shows a lack of uniformity in the use of LPFs and HPFs in the literature. In addition, these studies have reported a wider range of mean values for the latencies (8-12 msec for $\mathrm{n} 1$ latency and 13-17 msec for p1 latency) and amplitude $(3-10 \mu \mathrm{V})$ of oVEMP even among healthy individuals. This makes it difficult for the clinicians to use one of these values as normative for comparing against the pathological responses. Although a large range of filter sets have been used, there is limited experimental evidence to support one of these as the optimum or best filter set for eliciting air conduction tone burst-evoked oVEMP. The bandpass filter is used to eliminate the unwanted random electroencephalographic or electromyographic (EMG) activity that does not fall in the frequency spectrum of the desired response.

Recently, Wang et al (2013) studied the effect of changing response filters on ocular oVEMPs. In their study, oVEMP was recorded from 12 participants with normal auditory and vestibular system using various HPFs $(1,10$, and $100 \mathrm{~Hz})$ and LPFs $(500,1000$, and $2000 \mathrm{~Hz}$ ). Of these HPFs and LPFs, the LPF of 1000 $\mathrm{Hz}$ was kept constant with all the HPFs to form band-pass filters of 1-1000, 10-1000, and 100-1000 $\mathrm{Hz}$. They found the largest amplitude and $100 \%$ response rate for a band-pass filter of 1-1000 Hz. Furthermore, the HPF of $1 \mathrm{~Hz}$ was kept constant with all the LPFs to form band-pass filters of 1-500, 1-1000, and 1-2000 $\mathrm{Hz}$. For the variations in the LPF, the authors reported no significant difference between the response filter conditions. Based on these results, the authors suggested that $1-1000 \mathrm{~Hz}$ was optimum for recording oVEMP. The conclusions drawn in the study are based on a very small sample size $(\mathrm{N}=12)$, although the power spectrum analysis, which showed almost no energy beyond 1000 $\mathrm{Hz}$ in oVEMP responses, did substantiate their findings. Furthermore, the study did not compare the other frequently used filter sets in the literature.

Analyzing the response energy across frequency domain usually provides the basis for selecting a bandpass filter for a particular type of evoked potential recording. However, there is limited information on energy distribution across frequencies in case of oVEMP, which might have resulted in the use of a wide range of band-pass filters for recording oVEMP in the abovementioned studies. Furthermore, studies on oVEMP in the elderly have shown reduced response rates with advancing age (Nguyen et al, 2010). However, it is not known whether or not the interaction between a less appropriate band-pass filter and age could have yielded poorer response rates in elderly individuals. Hence, the present study attempted to study the effects of response filter sets on oVEMP elicited by air conduction tone bursts of $500 \mathrm{~Hz}$ and identify the optimum response filter for clinical recording of oVEMP.

\section{METHODS}

$\mathrm{T}$ he study was performed in two phases. Phase I was performed to identify the optimum band-pass filter for clinical recording of oVEMP, and it was performed using only the young adult population. Phase II was performed to investigate whether the optimum filter set could improve the chances of oVEMP detection among older adults with inherently reduced oVEMP amplitude and lower response rate. The study was approved by the biobehavioral research ethics committee of the institute.

\section{Participants}

Phase I of the study incorporated 150 ( 75 right and 75 left) ears of 150 individuals with normal auditory and vestibular systems in the age range of 18-35 years (mean $=22.7$ years, standard deviation $=3.9$ years, 
76 males and 74 females) after obtaining the informed written consents. The side (left or right) was chosen based on numbering. The odd-numbered participants underwent left ear acoustic stimulation, whereas even-numbered ones had their right ears stimulated for eliciting oVEMP. The normalcy of the auditory system was ensured through normal results on a battery of audiological tests including pure tone audiometry (four frequency pure tone average $\leq 15 \mathrm{~dB} \mathrm{HL}$ ), immittance evaluation using $226-\mathrm{Hz}$ probe tone (A type tympanograms [single-peaked tympanogram with admittance at tympanic membrane between 0.5 and $1.75 \mathrm{mmho}$, peak pressure between +60 and $-100 \mathrm{daPa}$, and tympanometric width $<100 \mathrm{daPa}$ ] with the presence of ipsilateral and contralateral acoustic reflexes below $100 \mathrm{~dB}$ HL for 500, 1000 , and $2000 \mathrm{~Hz}$ tonal stimuli), transient evoked otoacoustic emissions using click stimuli (signal-to-noise ratio [SNR] $>3 \mathrm{~dB}$ ) and dual channel click-evoked auditory brainstem response using 11.1 and $90.1 \mathrm{~Hz}$ stimulus repetition rates (inter-aural latency difference of $\leq 0.2 \mathrm{msec}$, inter-peak latency difference of $\leq 2.0 \mathrm{msec}$ for waves I-III and III-V and $\leq 4.0 \mathrm{msec}$ for waves I-V, and the amplitude ratio of $V / I \geq 0.5$ ). The vestibular well-being of the participants was ensured through normal results on behavioral balance assessment using the Fukuda stepping test $\left(<45^{\circ}\right.$ deviation in either direction from starting point), Romberg test (no sway in any direction), tandem gait test (able to walk heel-to-toe on an imaginary straight line for $10 \mathrm{~m}$ without losing balance), and past-pointing test (no tremors and undershooting or overshooting of the target on task involving alternate touching of clinician's finger and his/her nose tip) and objective assessment using video head impulse test (vestibulo-ocular reflex gain $>0.8$ and asymmetry of vestibulo-ocular reflex gain $<20 \%$ for all semicircular canals). In addition, a structured case history was obtained from the participants to ensure no history of any otological, vestibular, or neurological problems.

Phase II of the study incorporated ten healthy individuals (five males and five females) in the age range of 60-70 years who had bilaterally absent oVEMP when using a band-pass filter of 1-1000 Hz. In addition, they did not have history of obvious vestibular diseases such as Meniere's disease, benign paroxysmal positional vertigo, and vestibular neuritis/labyrinthitis, the negative results about which was confirmed through an otorhinolaryngology evaluation by an experienced otorhinolaryngologist. Furthermore, they did not have any neurological disorders, and this was ensured through a neurological opinion.

\section{Procedure}

Biologic Navigator Pro auditory evoked potential unit (version 7.2.1; Natus Medical Incorporated, Mundelein, IL) was used to acquire oVEMP from all participants.
The testing was performed in an acoustically treated room with ambient noise levels well within the acceptable levels for audiometric rooms (ANSI, 1991). The recording sites were cleaned with a commercially available abrasive gel to obtain absolute and interelectrode impedance below 5 and $2 \mathrm{k} \Omega$, respectively. The electrodes were placed using adequate amount of commercially available conduction paste and secured in place with adhesive tape used for surgeries. This electrode positioning used in the present study is similar to those used previously (Chihara et al, 2007; Singh and Barman, 2013; 2014; 2015). The noninverting electrode was placed on the cheek at about $1 \mathrm{~cm}$ below the center of the lower eyelid. The inverting electrode was placed directly below the noninverting electrode with a distance of $2 \mathrm{~cm}$ separating them. The ground electrode was positioned on the center of the forehead.

The stimulus and acquisition parameters described by previous studies, except filter setting, were replicated for the acquisition of oVEMP (Chihara et al, 2007; Rosengren et al, 2009; Wang et al, 2009; Singh and Barman, 2013; 2014; 2015). Single-channel recording was performed from the electrodes placed on the side contralateral to the stimulus ear as contralateral oVEMP was shown to be larger in amplitude than the ipsilateral one (Singh et al, 2015). The participants were instructed to elevate their gaze by $30^{\circ}$ in the midline to tense the inferior oblique muscle and increase its proximity to the surface during recording (Govender et al, 2009; Murnane et al, 2011; Rosengren et al, 2013). The accuracy and consistency of $30^{\circ}$ upward gaze in the midline was ascertained through ensuring the same head position before starting each rung and asking the participant to maintain gaze at the marker placed on the wall at that angle. Alternating polarity of $500-\mathrm{Hz}$ tone bursts, ramped using $1 \mathrm{msec}$ rise/fall time and 2 msec plateau time, were delivered at an intensity of 125-dB peSPL through the standard insert earphones SINSER-012 of Biologic Navigator Pro evoked potential system. The repetition rate used was $5.1 \mathrm{~Hz}$ because this rate has been found to be most efficient in evoking oVEMP by virtue of producing largest SNR, least amount of inter individual variations, and highest efficiency (Singh et al, 2014). Two hundred sweeps of EMG activity were recorded using an epoch of $64 \mathrm{msec}$, which included a $10.5 \mathrm{msec}$ prestimulus baseline recording. The differential signal was amplified 30,000 times, band-pass filtered, and then digitized at a rate of $8000 \mathrm{~Hz}$. Analog filter cutoffs (12 dB/octave filter slopes) were varied systematically. The band-pass filters included the low-pass cutoff frequencies of 500 , $700,1000,1500,2000$, and $3000 \mathrm{~Hz}$ and the high-pass cutoff frequencies of $0.1,1,10$, and $30 \mathrm{~Hz}$, in all possible combinations to form band-pass filters. These filter settings have been selected because these are the cutoffs that have most often been used in the studies on oVEMP 
in literature (Chihara et al, 2009; Nguyen et al, 2010; Murnane et al, 2011; Piker et al, 2011; Seo et al, 2013; Singh and Barman, 2013; Wang et al, 2013; Jerin et al, 2014; Singh and Barman, 2014; 2015). Furthermore, single runs were used per band-pass filter in each individual unless responses were below $2 \mu \mathrm{V}$, in which case they were replicated. The order of band-pass filter use was pseudo-random to avoid adulteration of the findings by the order of filter setting used. Recordings for all band-pass filters were performed in the same session, albeit with gaps of 1 min after every second recording or as desired by the participant to avoid muscle strain and involuntary eye blinks. Single-session recording was chosen over multisession recording option to counter against confounds due to slight changes in electrode impedance or location between the sessions. Participants were seated on an armed reclining chair with a head rest cushion to ensure comfort during recording of responses. During the 40-sec interval when oVEMP response acquisition was in progress, they were asked to avoid eye blinks. They were also instructed to avoid any movements of the limbs, torso, or any other parts of the body. These measures helped guard against muscle artifacts adulterating the true oVEMP response.

\section{The Power Spectrum Analysis of oVEMP Waveforms}

The power spectrum analysis was carried out to investigate the energy content in the oVEMP response waveform across the frequencies. For this, a MATLAB program (The MathWorks, Incorp., Bangalore, India) was used. The response corresponding to the widest filter set used in the study $(0.1-3000 \mathrm{~Hz})$ was used for this purpose. Power spectral density of the data was analyzed by using Welch modified periodogram method. Here, the signal was divided into eight non-overlapping windows with a Hanning taper. The eight windows were then subjected to a 24,576-point fast Fourier transform and the spectral densities were averaged across the windows. This was then log-transformed to obtain the power spectral densities in $\mathrm{dB}$.

\section{Measures}

The waveforms were analyzed by two independent experienced audiologists working in the area of vestibular assessment using VEMPs. An oVEMP was deemed present when the waveform was biphasic and it contained a negative going peak (n1) at about $10 \mathrm{msec}$ (8-13 msec) followed by a positive going peak (p1) at about $15 \mathrm{msec}(13-18 \mathrm{msec})$, as these are mean and range values reported for oVEMP peaks in the literature (Chihara et al, 2007; Cheng et al, 2009; Wang et al, 2009; Welgampola et al, 2009; Nguyen et al, 2010; Park et al, 2010; Murnane et al, 2011; Piker et al, 2011; Rosengren et al, 2011; Winters et al, 2011; Taylor et al, 2012). The parameters analyzed were n1 latency, p1 latency, and peak-to-peak amplitude. The SNR was calculated from each waveform using MATLAB software using the following formula:

$$
\mathrm{SNR}=20 \log \left(\mathrm{RMS}_{\mathrm{ep}} / \mathrm{RMS}_{\mathrm{b}}\right),
$$

where "SNR" is the signal-to-noise ratio in $\mathrm{dB}$, "RMS $\mathrm{ep}_{\mathrm{e}}$ " is the root-mean-square of the oVEMP response in the time range of 8-20 msec, and " $\mathrm{RMS}_{\mathrm{b}}$ " is the root-meansquare of the prestimulus baseline. The interjudge reliability was high ( $\alpha \geq 0.92$ for every band-pass filter, Cronbach's alpha test) for peak identification and the interjudge agreement was also high for the presence/ absence of oVEMP $(K \geq 0.934$ for every band-pass filter, Kappa coefficient). There was disagreement between the judges only on two responses in the $30-$ to $500-\mathrm{Hz}$ band-pass filter and in this case, a third judge's opinion was considered; whichever response (present/absent) had two of the three judges' backing was considered. In both these cases, the third judge deemed the response to be present. For the remaining band-pass filters, there was $100 \%$ agreement between the two judges about the presence of response.

\section{Statistical Analyses}

Statistical analyses were performed using commercially available software, Statistical Package for Social Science (version 17.0; SPSS South Asia (P) Ltd, Bangalore, India). The comparison of each response parameter was achieved through separate two-way repeated measures analysis of variance (ANOVA) for LPFs and HPFs separately for each response parameter. In case of a significant interaction between the variables, the use of focused tests of main effects through the techniques involving ANOVA has been recommended by several researchers (Kirk, 1982; Rosnow and Rosenthal, 1989; Stevens, 1990; Rosnow and Rosenthal, 1991; Winer et al, 1991) and put to use by several studies of VEMP (Singh et al, 2014; Singh and Barman, 2015). Therefore, in the present study, separate oneway repeated measures ANOVAs were carried out for all LPFs under each HPF and all HPFs under each LPF in case of significant interaction between the two kinds of filters. Bonferroni adjusted multiple comparisons were used for pair-wise comparison between different LPF and HPF pairs, in case a significant main effect was observed on the repeated measures ANOVA.

\section{RESULTS}

\section{Phase I}

In phase I, oVEMPs were present in $100 \%$ of the ears, irrespective of the band-pass filter. The individual 
averaged and grand averaged waveforms obtained for different HPFs and LPFs are shown in Figure 1. The waveforms were analyzed along three oVEMP parameterslatencies, amplitude, and SNR. The results for each of these parameters are discussed separately under specific headings in the subsequent sections that follow.

\section{Effects of Response Filter Setting on Latencies of oVEMP}

The latencies of $\mathrm{n} 1$ and $\mathrm{p} 1$ peaks were obtained and these were subjected to descriptive statistics for obtaining mean and standard deviation. Table 1 shows the mean and standard deviation of $\mathrm{n} 1$ and $\mathrm{p} 1$ latencies of oVEMP for various HPF and LPF combinations.

Two-way repeated measures ANOVA for HPFs and LPFs was performed for $\mathrm{n} 1$ latency. The results revealed a significant main effect of $\mathrm{HPF}\left[F_{(3,447)}=\right.$ 29.64, $p<0.001]$ and LPF $\left[F_{(5,745)}=118.76, p<\right.$ $0.001]$ on $n 1$ latency of oVEMP. There was no significant interaction between HPFs and LPFs $\left[F_{(15,2235)}=1.50\right.$, $p=0.098$ ]. The Bonferroni adjusted multiple comparisons were performed for pair-wise comparisons between different HPFs and also LPFs, and the results revealed a significant reduction in $\mathrm{n} 1$ latencies with increase in HPFs $(p<0.01)$ and LPFs $(p<0.008)$.

In terms of the $\mathrm{p} 1$ peak of oVEMP, there was a significant main effect of HPFs $\left[F_{(3,447)}=233.09, p<\right.$ $0.001]$ and LPFs $\left[F_{(5,745)}=29.11, p<0.001\right]$ on the latencies. In addition, there was a significant interaction between HPFs and LPFs $\left[F_{(15,2235)}=11.47, p<0.001\right]$.
To resolve the interaction, focused tests of main effects involving separate one-way repeated measures ANOVAs for each HPF and LPF were taken up. There was a significant main effect of HPFs on p1 latency of oVEMP for LPFs of $500 \mathrm{~Hz}\left[F_{(3,447)}=76.04, p<0.001\right], 700 \mathrm{~Hz}$ $\left[F_{(3,447)}=82.82, p<0.001\right], 1000 \mathrm{~Hz}\left[F_{(3,447)}=79.36\right.$, $p<0.001], 1500 \mathrm{~Hz}\left[F_{(3,447)}=115.13, p<0.001\right]$, $2000 \mathrm{~Hz}\left[F_{(3,447)}=97.82, p<0.001\right]$, and $3000 \mathrm{~Hz}$ $\left[F_{(3,447)}=84.47, p<0.001\right]$. Bonferroni adjusted multiple comparisons at each of the LPFs revealed a significant difference in $\mathrm{p} 1$ latency between the HPFs $(p<0.01)$.

Continuing with the focused tests, one-way repeated measures ANOVA revealed a significant main effect of LPFs on p1 latency of oVEMP for HPFs of $0.1 \mathrm{~Hz}\left[F_{(5,745)}=\right.$ 7.69, $p<0.001], 1 \mathrm{~Hz}\left[F_{(5,745)}=26.30, p<0.001\right], 10 \mathrm{~Hz}$ $\left[F_{(5,745)}=11.83, p<0.001\right]$, and $30 \mathrm{~Hz}\left[F_{(5,745)}=20.91\right.$, $p<0.001]$. The Bonferroni adjusted multiple comparisons at each of the HPFs revealed a significant difference in $\mathrm{p} 1$ latency between the LPFs $(p<0.008)$.

\section{Effects of Response Filter Setting on Amplitude of oVEMP}

The peak-to-peak amplitudes were obtained from the response waveforms for each band-pass filter and subjected to descriptive statistics for obtaining mean and standard deviation. Table 2 shows the mean and standard deviation of peak-to-peak amplitude of oVEMP for various HPF and LPF combinations.

A two-way repeated measures ANOVA was performed to evaluate the effect of different HPFs and LPFs on

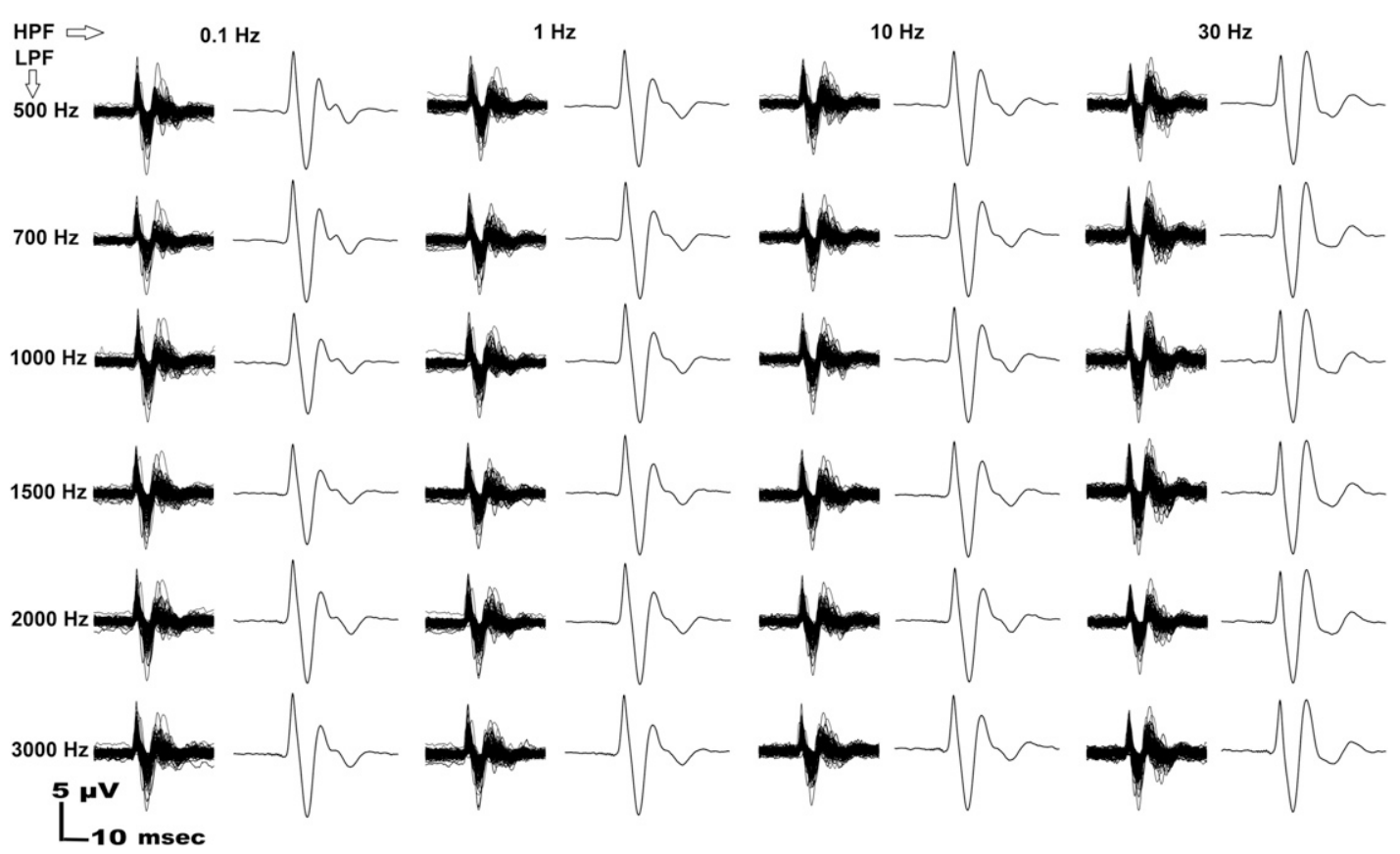

Figure 1. The individual-averaged and grand-averaged oVEMP waveforms acquired for various HPF and LPFs from 150 healthy individuals. The positivity is plotted in the downward direction. 
Table 1. Mean and Standard Deviation of $\mathrm{n} 1$ and $\mathrm{p} 1$ Latencies of oVEMP for Various HPF and LPF Combinations

\begin{tabular}{|c|c|c|c|c|c|c|c|c|}
\hline \multirow[b]{3}{*}{ LPFs (Hz) } & \multicolumn{8}{|c|}{ HPFs (Hz) } \\
\hline & \multicolumn{4}{|c|}{ n1 Latency (msec) } & \multicolumn{4}{|c|}{ p1 Latency (msec) } \\
\hline & 0.1 & 1 & 10 & 30 & 0.1 & 1 & 10 & 30 \\
\hline 500 & $10.59(0.50)$ & $10.73(0.57)$ & $10.63(0.53)$ & $10.52(0.56)$ & $15.65(0.92)$ & $16.28(0.85)$ & $15.87(0.86)$ & $15.50(0.90)$ \\
\hline 700 & $10.50(0.57)$ & $10.62(0.61)$ & $10.54(0.57)$ & $10.43(0.62)$ & $15.81(0.87)$ & $16.11(0.81)$ & $15.88(0.79)$ & $15.37(0.73)$ \\
\hline 1000 & $10.47(0.56)$ & $10.50(0.57)$ & $10.47(0.59)$ & $10.31(0.57)$ & $15.90(0.80)$ & $16.15(0.82)$ & $15.90(0.80)$ & $15.37(0.84)$ \\
\hline 1500 & $10.17(0.92)$ & $10.30(0.60)$ & $10.28(0.65)$ & $10.11(0.72)$ & $15.79(0.83)$ & $15.95(0.83)$ & $15.75(0.83)$ & $15.09(0.81)$ \\
\hline 2000 & $10.31(0.62)$ & $10.35(0.61)$ & $10.29(0.61)$ & $10.14(0.67)$ & $15.87(0.82)$ & $15.92(0.78)$ & $15.79(0.81)$ & $15.16(0.86)$ \\
\hline 3000 & $10.26(0.63)$ & $10.28(0.68)$ & $10.17(0.61)$ & $10.10(0.82)$ & $15.82(0.82)$ & $15.82(0.86)$ & $15.61(0.78)$ & $15.07(1.00)$ \\
\hline
\end{tabular}

Note: Standard deviation values are mentioned within brackets for each band-pass filter.

peak-to-peak amplitude of oVEMP. The results revealed a significant main effect of HPFs $\left[F_{(3,447)}=72.77, p<\right.$ $0.001]$ and LPFs $\left[F_{(5,745)}=4.54, p<0.001\right]$ on peakto-peak amplitude of oVEMP. Furthermore, there was a significant interaction between HPFs and LPFs $\left[F_{(15,2235)}=1.70, p=0.044\right]$. To resolve the interaction, focused tests of main effects involving separate one-way repeated measures ANOVAs for HPFs and LPFs were undertaken. There was a significant main effect of HPFs on peak-to-peak amplitude of oVEMP for LPFs of $500 \mathrm{~Hz}$ $\left[F_{(3,447)}=40.79, p<0.001\right], 700 \mathrm{~Hz}\left[F_{(3,447)}=33.44, p=\right.$ $0.000], 1000 \mathrm{~Hz}\left[F_{(3,447)}=49.80, p<0.001\right], 1500 \mathrm{~Hz}$ $\left[F_{(3,447)}=43.24, p<0.001\right], 2000 \mathrm{~Hz}\left[F_{(3,447)}=41.02\right.$, $p<0.001]$, and $3000 \mathrm{~Hz}\left[F_{(3,447)}=47.02, p<0.001\right]$. The Bonferroni adjusted multiple comparisons at each of the LPFs revealed a significant difference in peakto-peak amplitude between the HPFs $(p<0.01)$, except between some of the HPFs at each LPF. Specific outcome of the Bonferroni adjusted multiple comparisons between various HPFs at each LPF is shown in Table 2.
Furthermore, the focused test of main effects involving one-way repeated measures ANOVA for LPFs under each HPF revealed a significant main effect of LPFs on peak-topeak amplitude of oVEMP for HPFs of $0.1 \mathrm{~Hz}\left[F_{(5,745)}=\right.$ $5.93, p<0.001], 1 \mathrm{~Hz}\left[F_{(5,745)}=1.30, p=0.261\right], 10 \mathrm{~Hz}$ $\left[F_{(5,745)}=0.50, p=0.776\right]$, and $30 \mathrm{~Hz}\left[F_{(5,745)}=0.53, p=\right.$ 0.751]. The Bonferroni adjusted multiple comparisons at each of the HPF revealed a significant difference in peakto-peak amplitude between the LPFs ( $p<0.008)$, except for some of the LPFs at each HPF. Specific outcomes of the Bonferroni adjusted multiple comparisons between various LPFs at each of the HPFs are shown in Table 2.

\section{Effects of Response Filter Setting on SNR of oVEMP Waveforms}

In terms of the SNR of oVEMP, there was a significant main effect of HPFs $\left[F_{(3,447)}=7.71, p<0.001\right]$ but no significant main effect of LPFs $\left[F_{(5,745)}=0.27\right.$, $p=0.928]$ on the SNR. Furthermore, there was a

Table 2. Mean and Standard Deviation of Peak-to-Peak Amplitude and SNR of oVEMP for Various HPF and LPF Combinations

\begin{tabular}{|c|c|c|c|c|c|c|c|c|c|}
\hline \multirow[b]{3}{*}{ LPFs $(\mathrm{Hz})$} & \multicolumn{9}{|c|}{ HPFs (Hz) } \\
\hline & \multicolumn{5}{|c|}{ Peak-to-Peak Amplitude $(\mu \mathrm{V})$} & \multicolumn{4}{|c|}{ SNR } \\
\hline & 0.1 & 1 & 10 & 30 & & 0.1 & 1 & 10 & 30 \\
\hline 500 & $9.95(7.36)^{\star} \dagger \ddagger \S$ & $9.93(7.14) \S$ & $9.77(7.00) \S$ & $8.23(5.88) \|$ & $\Phi^{\star \star}$ & $28.63(12.52)$ & $27.76(13.15)$ & $31.42(12.29)$ & $28.36(11.74)$ \\
\hline 700 & $10.27(7.83)^{\star \star} \S$ & $9.79(6.92) \S$ & $9.62(6.73) \S$ & $8.34(5.77) \|$ & $\mid q^{\star \star}$ & $29.70(11.97)$ & $27.59(12.23)$ & $30.34(11.92)$ & $29.73(12.10)$ \\
\hline 1000 & $10.62(7.67) \dagger+\top^{\star \star}$ & $9.94(7.06) \| \S$ & $9.67(6.69) \| \S$ & $8.32(5.87) \|$ & $\mid q^{\star \star}$ & $30.25(13.17)$ & $28.43(12.18)$ & $30.28(11.74)$ & $28.89(11.01)$ \\
\hline 1500 & $10.59(7.62) \dagger \dagger^{* \star} \S$ & $10.08(7.34) \S$ & $9.76(6.97) \| \S$ & $8.41(5.89) \|$ & $\mid \Phi^{\star \star}$ & $31.39(13.50)$ & $27.12(12.30)$ & $29.32(12.20)$ & $29.21(11.91)$ \\
\hline 2000 & $10.36(7.50)^{\star \star} \S$ & $10.01(7.35) \S$ & $9.66(6.79) \| \S$ & $8.39(6.01) \|$ & $\mid \Phi^{\star \star}$ & $29.65(11.91)$ & $27.68(13.65)$ & $30.43(12.10)$ & $29.63(10.92)$ \\
\hline 3000 & $10.51(7.53) \dagger \dagger^{\star \star} \S$ & $10.15(7.36) \S$ & $9.69(6.83) \| \S$ & $8.37(5.79) \|$ & $\mid \Phi^{\star \star}$ & $30.91(12.52)$ & 27.09 (14.38) & $29.73(12.21)$ & $28.61(11.53)$ \\
\hline
\end{tabular}

Note: Standard deviation values are mentioned within brackets for each band-pass filter. All comparisons are at $p<0.01$ for HPF comparisons and at $p<0.008$ for LPF comparisons.

*Significantly different for comparison with 1000-Hz LPF within HPF within the same HPF. $\dagger$ Significantly different for comparison with $1500-\mathrm{Hz}$ LPF within HPF within the same HPF. $\ddagger$ Significantly different for comparison with $3000-\mathrm{Hz}$ LPF within HPF within the same HPF. §Significantly different for comparison with $30-\mathrm{Hz}$ HPF within HPF within the same LPF. \|Significantly different for comparison with $0.1-\mathrm{Hz}$ HPF within HPF within the same LPF. ISignificantly different for comparison with 1-Hz HPF within HPF within the same LPF.

${ }^{*}$ Significantly different for comparison with $10-\mathrm{Hz}$ HPF within HPF within the same LPF.

$\dagger+$ Significantly different for comparison with $500-\mathrm{Hz}$ LPF within HPF within the same HPF. 
significant interaction between HPFs and LPFs $\left[F_{(15,2235)}=1.74, p=0.012\right]$. To resolve this interaction, focused tests of main effects involving separate one-way repeated measures ANOVAs for each HPF and LPF were administered. There was a significant main effect of HPFs on SNR of the oVEMP response waveforms for LPFs of $500 \mathrm{~Hz}\left[F_{(3,447)}=4.56, p=0.004\right], 700 \mathrm{~Hz}$ $\left[F_{(3,447)}=3.04, p=0.028\right], 1500 \mathrm{~Hz}\left[F_{(3,447)}=6.15\right.$, $p<0.001], 2000 \mathrm{~Hz}\left[F_{(3,447)}=2.63, p=0.049\right]$, and $3000 \mathrm{~Hz}\left[F_{(3,447)}=5.75, p=0.001\right]$ but not of $1000 \mathrm{~Hz}$ $\left[F_{(3,447)}=1.94, p=0.122\right]$. The Bonferroni adjusted multiple comparisons at each of the LPF revealed a significant difference in SNR between very few pairs $(p<0.008)$. Furthermore, the focused tests for evaluating the effects of low-pass filtering on the SNR of the oVEMP response waveforms demonstrated a significant main effect of LPFs on the SNR for HPF of $0.1 \mathrm{~Hz}\left[F_{(5,745)}=2.44\right.$, $p=0.033]$ but not for $1 \mathrm{~Hz}\left[F_{(5,745)}=0.51, p=0.763\right]$, $10 \mathrm{~Hz}\left[F_{(5,745)}=1.57, p=0.164\right]$, and $30 \mathrm{~Hz}\left[F_{(5,745)}=\right.$ $1.00, p=0.416]$. However, the Bonferroni adjusted multiple comparisons at each of the HPF revealed no significant difference between the LPFs ( $p>0.008)$.

\section{The Power Spectrum Analysis of oVEMP Waveforms}

The power spectrum analysis was performed to investigate the energy content in the oVEMP response waveform across the frequencies. The major energy was observed up to $500 \mathrm{~Hz}$ with the peak at around $100 \mathrm{~Hz}$. There was no energy beyond $1000 \mathrm{~Hz}$. This was true for the responses from all 150 individuals. Figure 2 shows the power spectrum of the response waveform of oVEMP of an individual obtained for a broad filter setting of $0.1-3000 \mathrm{~Hz}$.

\section{Phase II}

In phase II, oVEMPs were obtained from 20 ears of ten older adults using 1 - to $1000-\mathrm{Hz}$ and $0.1-$ to $1000-\mathrm{Hz}$ bandpass filters. Of these, all (100\%) ears had absence of replicable oVEMP responses when using the 1- to $1000-\mathrm{Hz}$ band-pass filter, whereas oVEMPs were replicable and deemed present in two (10\%) of these ears on using the 0.1- to $1000-\mathrm{Hz}$ band-pass filter. These two ears were from two separate individuals. An example of such an occurrence is shown in Figure 3. The peak-to-peak amplitude was 1.6 and $1.35 \mu \mathrm{V}$ in these two ears for the 0.1 - to $1000-\mathrm{Hz}$ bandpass filter. Both these responses were deemed present by both the experienced judges (audiologists).

\section{DISCUSSION}

\section{Phase I (Young Adults)}

oVEMPs were obtained from all participants regardless of the recording band-pass filter employed. Our
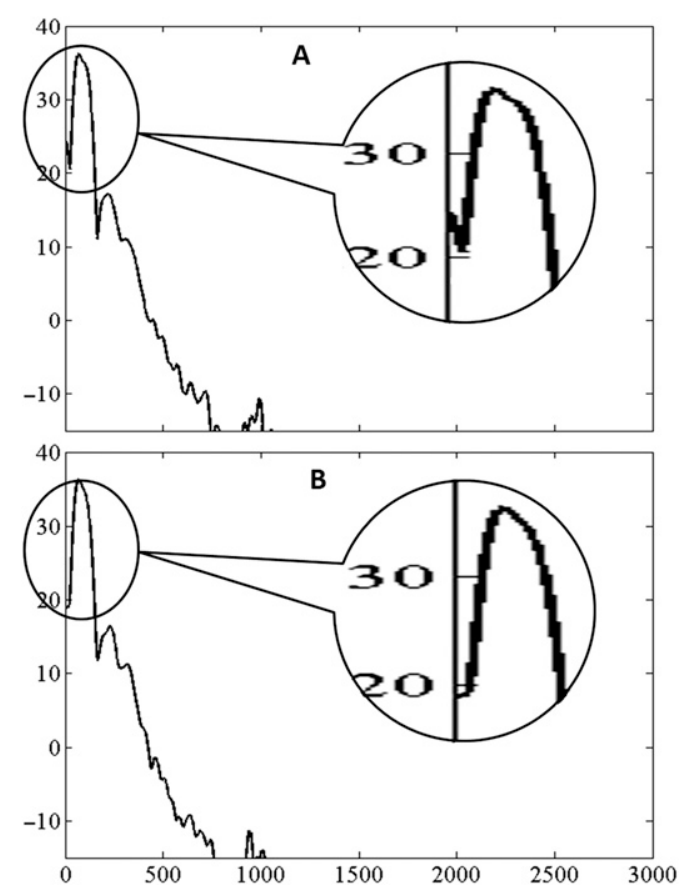

Figure 2. The power spectrum of super-averaged (grand-averaged) oVEMP waveforms obtained for filter settings of (A) $0.1-3000 \mathrm{~Hz}$ and (B) 1-1000 Hz. Both A and B show the magnified portion of lowfrequency region in the power spectra.

findings are similar to a previous report that was able to obtain a high response rate when employing bandpass filters of $1-500,1-1000$, and $10-1000 \mathrm{~Hz}$ (Wang et al, 2013). However, their response rate was decreased when using a 100-Hz HPF likely because of attenuation of a significant amount of oVEMP energy because peak oVEMP energy is centered near $100 \mathrm{~Hz}$. We restricted our $\mathrm{HPF}$ to a maximum of $30 \mathrm{~Hz}$, thereby ensuring against cutting-off of the peak of the response energy spectrum.

The results of the present study showed significant gradual shortening of latencies with increased HPF and LPF of the band-pass filter used for recording oVEMP. Although Wang et al (2013) observed a similar trend of reduction in latencies with increasing the LPFs and HPFs, the difference was significant only when the $\mathrm{HPF}$ was increased from 1 to $100 \mathrm{~Hz}$. Similar effects of changing the filter setting has been observed for other tone burst-evoked auditory evoked potentials such as auditory brainstem responses (Hyde, 1985). The reason behind reduction in the latencies with increasing the LPF and HPF could be the phase distortion that is introduced by the high-pass and low-pass components of a band-pass filter (Hyde, 1985). The LPF has been associated with the smoothening of the high-frequency components (Hyde, 1985). However, an HPF is believed to produce time lead components (negative delay) (Hyde, 1985). Furthermore, the HPF effect was shown to be more pronounced compared with the LPF. These factors 


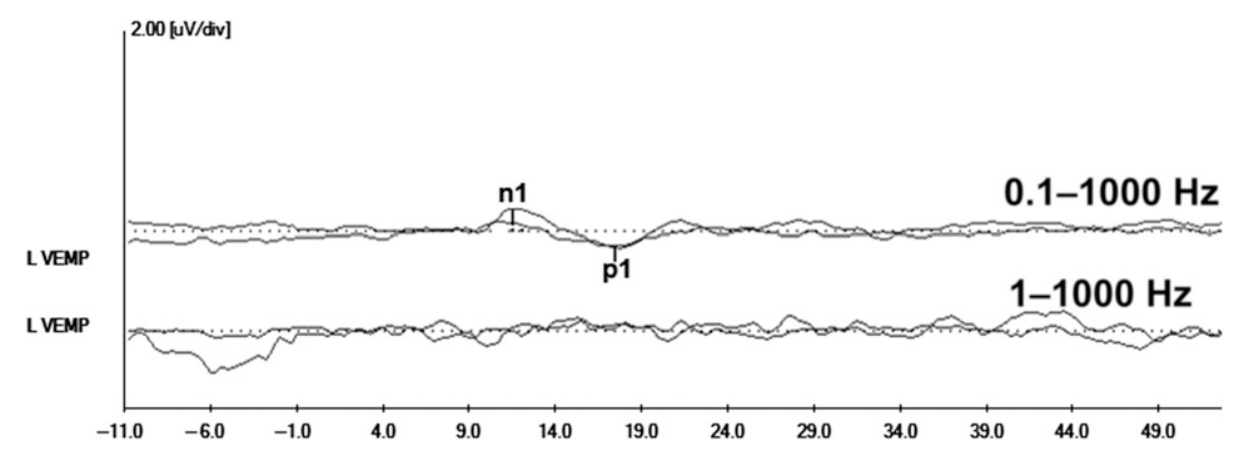

Figure 3. oVEMP waveforms recorded using $0.1-$ to $1000-\mathrm{Hz}$ and 1- to $1000-\mathrm{Hz}$ band-pass filters from a $65-$ year-old adult.

in cohesion might have caused gradual shortening of the peak latencies in the present study.

In terms of the peak-to-peak amplitude, the results of the present study revealed significant progressive reduction in amplitude with increase in HPF but not LPF. This is again in cohesion with the findings of Wang et al (2013). These findings (no significant change in amplitude with changes in LPFs) could be attributed to the frequency composition of the oVEMP response which revealed only a small amount of energy between $500 \mathrm{~Hz}$ and $1000 \mathrm{~Hz}$ and almost no energy above 1000 $\mathrm{Hz}$. Because the lowest LPF in the present study was $500 \mathrm{~Hz}$, changes in LPFs beyond $500 \mathrm{~Hz}$ did not significantly impact the amplitude, as it possibly did not alter energy content within the response waveforms. Furthermore, the studies on auditory evoked potentials have shown that an HPF is usually associated, depressing the amplitude of the given response, especially for responses containing predominantly low-frequency spectra, and introducing an artificial succeeding peak of opposite polarity for the use of narrow filters (Hyde, 1985), like the one used in the present study. This might be one of the reasons behind the finding of reduction in the peak-to-peak amplitude of oVEMP with increase in the cutoff frequency of the HPF. In addition, the major energy concentration in the power spectrum of the oVEMP waveforms (as revealed by the power spectrum analyses) is seen in the low-frequency region with a peak at about $100 \mathrm{~Hz}$, same as that found by Wang et al (2013) in their power spectrum analysis. This causes major changes in the response energy when the high-pass cutoff is progressively increased, which in turn will yield responses with progressively smaller amplitudes.

The results of the present study showed significantly better SNR for some of the HPFs; however, there was no pattern to such differences. In fact, most of the frequency pairs were not significantly different from each other. The differences might be attributed to the chance results. The lack of significant difference might be attributed to the attributes of an SNR measurement. SNR is the difference between the signal level and the amplitude of the noise floor. Signal is a relatively stable factor in case of oVEMP as it describes the peak-to-peak amplitude which can be reliably recorded over several recordings (Nguyen et al, 2010; Singh et al, 2011). However, noise, which in case of electrophysiological tests such as oVEMP would arise mainly from the physiological activities within the human body, is a random phenomenon as it is not linked to any stimulus (Dawson, 1950). This (physiological noise) is likely to vary between epochs and also from one individual to another (Dawson, 1950). The other sources of noise could include electrical noise in the environment, which, however, might not be random and, therefore, might not have contributed to these outcomes. Therefore, the difference between the response amplitude (signal), which is relatively constant, and noise would be less stable. This might have caused a lack of any pattern with variations in high-pass cutoff frequencies of the HPF.

An optimum filter set should be capable of producing responses in all healthy individuals and should produce largest amplitude and high SNRs. The power spectrum analysis of all responses in the present study showed the presence of energy only up to $1000 \mathrm{~Hz}$ and no energy beyond this. This means that using filters with low-pass cutoff above $1000 \mathrm{~Hz}$ will not alter the response amplitude, but extending beyond this could increase the chance of response adulteration by picking up high-frequency noise. Furthermore, use of 500 or $1000 \mathrm{~Hz}$ was found to reduce the response amplitude and SNR in the response to an extent, although not statistically significant. Therefore, $1000 \mathrm{~Hz}$, as also shown by Wang et al (2013), is a better choice for low-pass cutoff than all other frequencies used in the present study. When $1000 \mathrm{~Hz}$ was combined with $0.1 \mathrm{~Hz}$, it resulted in larger amplitude of oVEMP than 1, 10, and $30 \mathrm{~Hz}$ HPFs. Furthermore, use of a $0.1-$ to $1000-\mathrm{Hz}$ filter set produced higher SNR than 1-1000 and $30-1000 \mathrm{~Hz}$, whereas comparable values to $10-1000 \mathrm{~Hz}$ were obtained. Furthermore, the use of a 0.1 - to $1000-\mathrm{Hz}$ band-pass filter, by virtue of producing the largest amplitude, might enhance the chances of oVEMP detection even in older individuals with reduced muscle tone in whom the oVEMP might be otherwise difficult to detect (Nguyen et al, 2009). Therefore, the band-pass filter of 0.1-1000 $\mathrm{Hz}$, by virtue of producing the largest peak-to-peak 
amplitude and SNR, appears to be the optimum filter setting for the clinical recording of oVEMP. This is in disagreement with the findings of Wang et al (2013) who found $1-1000 \mathrm{~Hz}$ as the optimum band-pass filter for recording oVEMP. The differences between the studies could be attributed to the nonuse of $0.1 \mathrm{~Hz}$ as an HPF in the study by Wang et al (2013). It could be plausible that the use of $0.1 \mathrm{~Hz}$ as one of the HPF options might have probably shown $0.1-1000 \mathrm{~Hz}$, rather than $1-1000 \mathrm{~Hz}$, as the optimum filter setting for acquiring oVEMP, as was seen in the present study.

\section{Phase II (Older Adults)}

In phase II of the present study, 20 ears of older adults underwent oVEMP testing using the conventionally used 1- to $1000-\mathrm{Hz}$ band-pass filter and the one found optimum as a result of phase I of the study $(0.1-1000 \mathrm{~Hz})$. This was performed to investigate if indeed the use of 0.1 - to $1000-\mathrm{Hz}$ band-pass filters could improve the chances of oVEMP detection in the populations known to show low response rates. Whereas none of the 20 ears showed identifiable oVEMP response for 1- to $1000-\mathrm{Hz}$ filters, two ears showed low amplitude replicable oVEMPs when using 0.1- to $1000-\mathrm{Hz}$ bandpass filters. Therefore, the hypothesis that $0.1-$ to $1000-\mathrm{Hz}$ band-pass filters could improve the chances of detecting oVEMP compared with 1- to $1000-\mathrm{Hz}$ filters appears true. This (better response rate at $0.1-1000 \mathrm{~Hz}$ than $1-1000 \mathrm{~Hz}$ ) might be attributed to higher amplitude responses for the $0.1-$ to $1000-\mathrm{Hz}$ band-pass filter than the 1- to $1000-\mathrm{Hz}$ one, as was shown by the results of phase I of the present study. There could be a chance that errors in averaging introduced by an extremely low set HPF cutoff might have caused deflections in the oVEMP waveform, which was spuriously noted as responses in two elderly individual ears. Although there is no concrete way of categorically stating whether or not such an error occurred, a few steps could possibly tilt the decision in favor of them being actual responses rather than noises misidentified as responses. First, such noise could possibly affect a few recordings but not all. Therefore, if super-averaged or grand-averaged waveform spectrum shows significant energy below $1 \mathrm{~Hz}$, it could support the fact that the responses in two elderly ears were actual oVEMPs. Figures 3A and $B$ show power spectra of super-averaged waveforms obtained from phase I of the present study corresponding to $0.1-$ to $1000-\mathrm{Hz}$ and $1-$ to $1000-\mathrm{Hz}$ filters, respectively. It can be clearly noticed in the magnified images that there is significantly higher amount of energy in the extreme low-frequency part of the power spectrum (possibly $<1 \mathrm{~Hz}$ ) in Figure $3 \mathrm{~A}$ (which corresponds to the 0.1- to $1000-\mathrm{Hz}$ filter) than Figure 3B (which corresponds to the 1- to $1000-\mathrm{Hz}$ filter). Second, the characteristics of the obtained responses from the two elderly ears accurately fit the characteristics of a typical oVEMP response. These characteristics were as follows: (a) first, there is a negative peak followed by a positive peak; (b) the latency of the negative peak $(12.1 \mathrm{msec})$ and the positive peak ( $16.3 \mathrm{msec})$ was well within the expected latency range of oVEMP for the age group (Nguyen et al, 2010; Tseng et al, 2010); and repeated recording with changes in the epoch (epoch was decreased to $50 \mathrm{msec}$ and increased to $90 \mathrm{msec}$ ) showed repeatable outcomes to that found on the first instance. All these possibly are supportive enough for the two deflections in EMG found for the 0.1- to $1000-\mathrm{Hz}$ filter as an actual oVEMP response and not random noise being identified as a chance response.

The reason for the presence of response for $0.1-1000$ $\mathrm{Hz}$ despite the absence for the $1-$ to $1000-\mathrm{Hz}$ filter could be explained as follows. The smaller amplitude responses for the 1- to $1000-\mathrm{Hz}$ band-pass filter could have been so small that they were concealed among the EMG and other biological noises which prevented their detection. On the contrary, slightly larger amplitude responses for the 0.1 - to $1000-\mathrm{Hz}$ band-pass filter were possibly large enough to cause visibility beyond the random EMG noises and therefore got detected. This is a further testimony to the outcomes of phase I of the study which showed that $0.1-1000 \mathrm{~Hz}$ was a better band-pass filter than the 1- to $1000-\mathrm{Hz}$ one and should, therefore, be considered optimum for clinical recording of oVEMP. Similar better response detection could be a probability in some of the individuals suffering from clinical conditions such as Meniere's disease or benign paroxysmal positional vertigo (BPPV), which are known to result in amplitude reduction of oVEMP or cause its absence in several cases (Singh and Barman, 2015; 2016a,b; Singh and Apeksha, 2016), which, however, can be confirmed only after experimental evidence from future studies in this regard. Here, it would be important to mention a word of caution that two of 20 ears represent a small sample and require more controlled studies to further assert the findings. However, at this point in time, it does depict a promising result, provided future studies can show complementary results in this regard.

\section{CONCLUSIONS}

$\mathrm{T}$ he present study showed that oVEMP latency significantly decreased as the LPF and/or HPF increased. In addition, oVEMP amplitude significantly decreased as the HPF increased. The largest oVEMP amplitude was recorded using a band-pass filter of $0.1-1000 \mathrm{~Hz}$ and these recordings had an SNR that was similar to those with other band-pass filters. The study also showed that chances of detecting oVEMP improved when using $0.1-$ to $1000-\mathrm{Hz}$ band-pass filters over 1- to $1000-\mathrm{Hz}$ filters and, therefore, the use of 
0.1 - to $1000-\mathrm{Hz}$ band-pass filters could increase the chances of response detection, thereby increasing the diagnostic efficacy of oVEMP in difficult cases such as older adults. Consequently, our findings suggest that 0.1- to $1000-\mathrm{Hz}$ band-pass filters are optimal for clinical recordings of oVEMP.

Acknowledgments. We thank the director, All India Institute of Speech and Hearing, Mysore, and the HOD Audiology for granting permission to carry out the study, and the participants of the study for their participation and kind cooperation throughout the course of the study.

\section{REFERENCES}

American National Standards Institute (ANSI). (1991) Criteria for Maximum Permissible Ambient Noise during Audiometric Testing. ANSI S3.1. New York, NY: ANSI.

Cacace AT, Shy M, Satya-Murti S. (1980) Brainstem auditory evoked potentials: a comparison of two high-frequency filter settings. Neurology 30(7, Pt 1):765-767.

Cheng PW, Chen CC, Wang SJ, Young YH. (2009) Acoustic, mechanical and galvanic stimulation modes elicit ocular vestibularevoked myogenic potentials. Clin Neurophysiol 120:1841-1844.

Cheng YL, Wu HJ, Lee GS. (2012) Effects of plateau time and ramp time on vestibular evoked myogenic potentials. $J$ Vestib Res 22(1):33-39.

Chihara Y, Iwasaki S, Fujimoto C, Ushio M, Yamasoba T, Murofushi T. (2009) Frequency tuning properties of ocular vestibular evoked myogenic potentials. Neuroreport 20:1491-1495.

Chihara Y, Iwasaki S, Ushio M, Murofushi T. (2007) Vestibularevoked extraocular potentials by air conducted sound: another clinical test for vestibular function. Clin Neurophysiol 118: 2745-2751.

Colebatch JG, Halmagyi GM. (1992) Vestibular evoked potentials in human neck muscles before and after unilateral vestibular deafferentation. Neurology 42(8):1635-1636.

Curthoys IS. (2010) A critical review of the neurophysiological evidence underlying clinical vestibular testing using sound, vibration and galvanic stimuli. Clin Neurophysiol 121(2):132-144.

Dawson GD. (1950) Cerebral responses to nerve stimulation in man. Br Med Bull 6:326-329.

El-Mahallawi TH, Gabr TA, Hamada SM, Monem SEA. (2012) Vestibular evoked myogenic potentials (VEMPs) with different recording procedures. Egyp J Ear Nose Throat Allied Sci 13:113-120.

Goodin DS, Aminoff MJ, Chequer RS. (1992) Effect of different high-pass filters on the longlatency event-related auditory evoked potentials in human subjects and individuals infected with the human immunodeficiency virus. J Clin Neurophisiol 9(1):97-104.

Govender S, Rosengren SM, Colebatch JG. (2009) The effect of gaze direction on the ocular vestibular evoked myogenic potential produced by air-conducted sound. Clin Neurophysiol 120(7): $1386-1391$.

Gozke E, Erdal N, Ozkarakas H. (2010) Ocular vestibular evoked myogenic potentials in patients with migraine. Acta Neurol Belg 110:321-324.
Hyde ML. (1985) Instrumentation and signal processing. In: Jacobson T, ed. The Auditory Brainstem Response. London, UK: Taylor \& Francis Ltd., pp. 33-48.

Jerin C, Berman A, Krause E, Ertl-Wagner B, Gurov R. (2014) Ocular vestibular evoked myogenic potential frequency tuning in certain Meniere's disease. Hear Res 310:54-59.

Kantner C, Hapfelmeier A, Drexl M, Gurkov R. (2014) The effects of rise/fall time and plateau time on ocular vestibular evoked myogenic potentials. Eur Arch Otorhinolaryngol 271(9): 2401-2407.

Kirk RE. (1982) Experimental Design Procedures for the Behavioral Sciences. 2nd ed. Belmont, CA: Brooks/Cole.

Lim LJ, Dennis DL, Govender S, Colebatch JG. (2013) Differential effects of duration for ocular and cervical vestibular evoked myogenic potentials evoked by air- and bone-conducted stimuli. Exp Brain Res 224(3):437-445.

Murnane OD, Akin FW, Kelly JK, Byrd S. (2011) Effects of stimulus and recording parameters on the air conduction ocular vestibular evoked myogenic potential. J Am Acad Audiol 22: $469-480$.

Nguyen KD, Minor LB, Santina CCD, Carey JP. (2009) Vestibular function and vertigo control after intratympanic gentamicin for Meniere's disease. Audiol Neurotol 14(6):361-372.

Nguyen KD, Welgampola MS, Carey JP. (2010) Test-retest reliability and age-related characteristics of the ocular and cervical vestibular evoked myogenic potential tests. Otol Neurotol 31(5): 793-802.

Park HJ, Lee IS, Shin JE, Lee YJ, Park MS. (2010) Frequency tuning characteristics of cervical and ocular vestibular evoked myogenic potentials induced by air-conducted tone bursts. Clin Neurophysiol 121(1):85-89.

Piker EG, Jacobson GP, McCaslin DL, Hood LJ. (2011) Normal characteristics of ocular vestibular evoked myogenic potential. $J$ Am Acad Audiol 22:222-230.

Rosengren SM, Colebatch JG, Straumann DS, Weber KP. (2013) Why do oVEMPs become larger when you look up? Explaining the effect of gaze elevation on the ocular vestibular evoked myogenic potential. Clin Neurophysiol 124:785-791.

Rosengren SM, Govender S, Colebatch JG. (2011) Ocular and cervical vestibular evoked myogenic potentials produced by air- and bone-conducted stimuli: comparative properties and effects of age. Clin Neurophysiol 122:2282-2289.

Rosengren SM, Jombik P, Halmagyi GM, Colebatch JG. (2009) Galvanic ocular vestibular evoked myogenic potentials provide new insight into vestibulo-ocular reflexes and unilateral vestibular loss. Clin Neurophysiol 120:569-580.

Rosengren SM, Todd NPM, Colebatch JG. (2005) Vestibularevoked extraocular potentials produced by stimulation with boneconducted sound. Clin Neurophysiol 116:1938-1948.

Rosnow R, Rosenthal R. (1991) If you're looking at the cell means, you're not looking at only the interaction (unless all main effects are zero). Psychol Bull 110(3):574-576.

Rosnow R, Rosenthal R. (1989) Definition and interpretation of interaction effects. Psychol Bull 105(1):143-146.

Sandhu JS, George SR, Rea PA. (2013) The effect of electrode positioning on the ocular vestibular evoked myogenic potential to air-conducted sound. Clin Neurophysiol 124(6):1232-1236. 
Seo T, Saka N, Ohta S, Sakagami M. (2013) Detection of utricular dysfunction using ocular vestibular evoked myogenic potential in patients with benign paroxysmal positional vertigo. Neurosci Lett $550: 12-16$.

Singh NK, Apeksha K. (2016) Efficacy of cervical and ocular vestibular evoked myogenic potentials in evaluation of benign paroxysmal positional vertigo of posterior semicircular canal. Eur Arch Otorhinolaryngol 273(9):2523-2532.

Singh NK, Barman A. (2013) Characterizing the frequency tuning property of air-conduction ocular evoked myogenic potential in healthy individual. Int $J$ Audiol 52:849-854.

Singh NK, Barman A. (2014) Characterizing the effects of frequency on parameters of short tone bursts induced ocular vestibular evoked myogenic potentials. J Indian Speech Lang Hearing Assoc 28(1):1-9.

Singh NK, Barman A. (2015) Efficacy of ocular vestibular-evoked myogenic potential in identifying posterior semicircular canal benign paroxysmal positional vertigo. Ear Hear 36(2):261-268.

Singh NK, Barman A. (2016a) Frequency-amplitude ratio of ocular vestibular evoked myogenic potentials for detecting Meniere's disease: a preliminary investigation. Ear Hear 37(3):365-373.

Singh NK, Barman A. (2016b) Utility of the frequency tuning measure of oVEMP in differentiating Meniere's disease from BPPV. $J$ Am Acad Audiol 27(9):764-777.

Singh NK, Kadisonga P, Ashitha P. (2014) Optimizing stimulus repetition rate for recording ocular vestibular evoked myogenic potential elicited by air-conduction tone bursts of $500 \mathrm{~Hz}$. Audiol Res 4(88):14-20.

Singh NK, Kumar P, Aparna TH, Barman A. (2014) Rise/fall and plateau time optimization for cervical vestibular-evoked myogenic potential elicited by short tone bursts of $500 \mathrm{~Hz}$. Int $J$ Audiol 53(7): 490-496.

Singh NK, Sarda S, Sinha S, Tamsekar SS. (2011) Test retest reliability of ocular vestibular evoked myogenic potentials. J All India Inst Speech Hear 30:207-210.

Singh NK, Valappil N, Mithlaj JA. (2015) Response rates and testretest reliability of ipsilateral and contralateral ocular vestibular evoked myogenic potential in healthy adults. Hear Bal Commun 13:126-133.

Stevens J. (1990) Intermediate Statistics: a Modern Approach. Hillsdale, NJ: Lawrence Erlbaum Associates Inc.

Taylor RL, Bradshaw AP, Halmagyi GM, Welgampola MS. (2012) Tuning characteristics of ocular and cervical vestibular evoked myogenic potentials in intact and dehiscent ears. Audiol Neurotol $17: 207-218$.

Tseng CL, Chou CH, Young YH. (2010) Aging effect on the ocular vestibular-evoked myogenic potentials. Otol Neurotol 31: 959-963.

Todd NPM, Rosengren SM, Aw ST, Colebatch JG. (2007) Ocular vestibular evoked myogenic potentials (oVEMPs) produced by air- and bone-conducted sound. Clin Neurophysiol 118:381-390.

Walther LE, Blodow A. (2013) Ocular vestibular evoked myogenic potential to air conducted sound stimulation and video head impulse test in acute vestibular neuritis. Otol Neurotol 34: 1084-1089.

Wang SJ, Jaw FS, Young YH. (2009) Ocular vestibular-evoked myogenic potentials elicited from monaural versus binaural acoustic stimulation. Clin Neurophysiol 120:420-423.

Wang SJ, Jaw FS, Young YH. (2013) Optimizing the bandpass filter for acoustic stimuli in recording ocular vestibular-evoked myogenic potentials. Neurosci Lett 542:12-16.

Welgampola MS, Migliaccio AM, Myrie OA, Minor LB, Carey JP. (2009) The human soundevoked vestibule-ocular reflex and its electromyographic correlate. Clin Neurophysiol 120(1):158-166.

Winer BJ, Brown DR, Michels KM. (1991) Statistical Principles in Experimental Design. 3rd ed. New York, NY: McGraw-Hill.

Winters SM, Capschroer T, Grolman W, Klis SF. (2011) Ocular vestibular evoked myogenic potentials in response to airconducted sound in Meniere's disease. Otol Neurotol 32: $1273-1280$

Zhou G, Cox LC. (2004) Vestibular evoked myogenic potentials: history and overview. Am J Audiol 13(2):135-143. 